\title{
Methods for Applying Numerical Maps of Ionospheric Characteristics
}

\author{
William B. Jones and Roger M. Gallet
}

\author{
Contribution From Central Radio Propagation Laboratory, National Bureau of Standards, \\ Boulder, Colo.
}

(Received May 22, 1962)

\begin{abstract}
A solution has recently been given [Jones and Gallet, 1962] to the problem of representing the complex variations of ionospheric characteristics on a worldwide scale, including their diurnal variation, by numerical analysis of ionospheric data as measured at the stations, without prior hand operations. Whereas the paper referred to above deals with "how to make numerical maps," the present paper is concerned with "how to use a numerical map" once it has been obtained. Included are (1) a precise definition of a numerical map, (2) efficient computing procedures for applying numerical maps, including a general method for computing worldwide (or polar) contour maps in either universal time or local mean time, and (3) a large selection of graphical illustrations computed automatically from a numerical map.
\end{abstract}

\section{Introduction}

In a recent paper by Jones and Gallet [1962] a solution was given to the problem of representing the complex properties of ionospheric characteristics on a worldwide scale, including their diurnal variation, by numerical analysis of ionospheric data as measured at the stations, without prior hand operation. ${ }^{1}$ The solution referred to above is genera] enough to be applied to any ionospheric characteristic, and, in fact, has already been applied to the following: the critical frequency $\left(f_{0} F_{2}\right)$, the $3000-\mathrm{km}$ maximum usable frequency factor $\left(F_{2}-\mathrm{M} 3000\right)$, the maximum electron density $\left(N_{\max }\right)$, the height of $\Lambda_{\max }\left(H_{\max }\right)$, and the quarter thickness of a layer (SCAT) [see Wright, Wescott, and Brown, 1961]. With only slight modification, the methods could also include variations with height above the surface of the earth.

Ionospheric representations (or maps) obtained from this solution are given in a numerical form and are therefore referred to as numerical maps (sec. 2). Whereas the first reference [Jones and Gallet, 1962] deals with "how to make numerical maps," the present paper is concerned with "how to use a numerical map" once it has been obtained.

A discussion of methods for applying numerical maps is given in section 3 . Included are efficient computing procedures for evaluating a numerical map and a general method for computing worldcontour maps in either universal time or local mean time. A wide selection of such graphical representations is given in section 4. Graphical displays of these types are needed for solving problems of $\mathrm{HF}$ radio propagation by manual operations and, moreover, serve a useful purpose in describing the complex properties of the ionosphere. One should recognize,

1 For a brief summary of the problem and solution, the reader can refer to [Jones and Gallet, 1960]. however, that when electronic computing equipment is available, it is more accurate and usually more efficient to use numerical maps directly within the computer than to read and interpolate from contour maps.

As a means of illustration, the numerical map used in the present paper is for the monthly median of $f_{0} F_{2}$, September 1958. The types of illustrations given here could also be given for any other characteristic, and for predicted numerical maps for future months, since these maps have exactly the same mathematical form. We begin in the following section by giving a precise definition of a numerical map.

\section{Numerical Maps $\Gamma(\lambda, \theta, t)$}

The term, numerical map, is used to denote a function $\Gamma(\lambda, \theta, t)$ of three variables, latitude $(\lambda)$, longitude $(\theta)$, and time $(t)$, which represents an ionospheric characteristic, including its diurnal variation, on a worldwide scale. In order to apply such a function once it has been obtained, one must know its exact form and the range of definition of each independent variable. We begin with the latter:

$$
\begin{aligned}
& \lambda=\text { geographic latitude: } \quad-90^{\circ} \leqq \lambda \leqq 90^{\circ} \text {, } \\
& \theta=\text { geographic longitude: } \quad 0^{\circ} \leqq \theta \leqq 360^{\circ} \text {, }
\end{aligned}
$$$$
\text { ( } \theta \text { in degrees east of Greenwich) }
$$$$
t=\text { local mean hour angle: }-180^{\circ} \leqq t \leqq 180^{\circ} \text {, }
$$

$t=$ local mean hour angle: $-180^{\circ} \leqq t \leqq 180^{\circ}$, $t=\left(15 h-180^{\circ}\right)$, where $h=$ local mean time (LM'T) in hours.

For example, at noon LMT, $h=12$ and $t=0^{\circ}$. Universal time (UT) can therefore be introduced by means of the relation

$$
T=t-\theta, \quad-180^{\circ} \leqq T \leqq 180^{\circ},
$$


where $T$ is the universal hour angle.

The general form of $\Gamma(\lambda, \theta, t)$ is a "Fourier" time series

$\Gamma(\lambda, \theta, t)=a_{0}(\lambda, \theta)+\sum_{j=1}^{H}\left[a_{j}(\lambda, \theta) \cos j t+b_{j}(\lambda, \theta) \sin j t\right]$

where $H$ denotes the number of harmonics retained for the diurnal variation and where the "Fourier" coefficients $a_{j}(\lambda, \theta)$ and $b_{j}(\lambda, \theta)$ vary with the geographic coordinates. ${ }^{2}$ Such variations are represented in the form

$$
\begin{array}{ll}
a_{j}(\lambda, \theta)=\sum_{k=0}^{K} D_{2 j, k} G_{k}(\lambda, \theta) & j=0,1, \ldots, H, \\
b_{j}(\lambda, \theta)=\sum_{k=0}^{K} D_{2 j-1, k} G_{k}(\lambda, \theta) & j=1,2, \ldots, H,
\end{array}
$$

where the geographic functions $G_{k}(\lambda, \theta)$ are given in table 1 . As can be seen the functions $G_{k}(\lambda, \theta)$ consist of three main groups, each having different types of longitudinal terms present. Thus a specific set of the functions $G_{k}(\lambda, \theta)$ is specified by assigning the values $q_{0}, q_{1}$, and $q_{2}$ which are, respectively, the highest powers of $\sin \lambda$ for terms involving (1) no longitudinal variation, (2) first order longitudinal variation, and (3) second-order longitudinal variation. Equivalently, one could also specify the values $k_{0}, k_{1}$, and $k_{2}=K$ taken by the index $k$ at the end of each of the three groups of the $G_{i i}(\lambda, \theta)$, respectively. These values are related to the $q$ values by

$$
k_{0}=q_{0} \quad \text { and } \quad k_{r}=k_{r-1}+2\left(q_{r}+1\right) \quad \text { for } \quad r=1,2 .
$$

A numerical map $\Gamma(\lambda, \theta, t)$ can therefore be written more explicitly in the form

$$
\begin{aligned}
\Gamma(\lambda, \theta, t)=\sum_{k=0}^{K} D_{0, k} G_{k}(\lambda, \theta) \\
+\sum_{j=1}^{H}\left\{\left[\sum_{k=0}^{K} D_{2 j, k} G_{k}(\lambda, \theta)\right] \cos j t\right. \\
\left.+\left[\sum_{k=0}^{K} D_{2 j-1, k} G_{k}(\lambda, \theta)\right] \sin j t\right\}
\end{aligned}
$$

which is defined by a relatively small table of coefficients $D_{s k}$. An example of such a set of coefficients is given in table 2 for the characteristic $f_{0} F_{2}$ monthly median for September 1958 . As can be seen from table 2, the number of harmonics retained is $H=8$ and the parameters required to specify the functions $G_{k}(\lambda, \theta)$ are given by $k_{0}=11$, $k_{1}=37$, and $k_{2}=K=45$. Hence from (7) we have for the highest powers of $\sin \lambda$ in each of the three groups of the $G_{k}(\lambda, \theta), q_{0}=11, q_{1}=12$, and $q_{2}=3$.

\begin{tabular}{|c|c|c|c|c|c|}
\hline \multirow{2}{*}{\multicolumn{2}{|c|}{$\begin{array}{l}\text { Main latitudinal } \\
\text { variation }\end{array}$}} & \multicolumn{4}{|c|}{ Mixed latitudinal and longitudinal variation } \\
\hline & & \multicolumn{2}{|c|}{ First order in longitude } & \multicolumn{2}{|c|}{ Second order in longitude } \\
\hline$k$ & $G_{k}(\lambda, \theta)$ & $k$ & $G_{k}(\lambda, \theta)$ & $k$ & $G_{k}(\lambda, \theta)$ \\
\hline $\begin{array}{l}0 \\
1 \\
2\end{array}$ & $\begin{array}{c}1 \\
\sin \lambda \\
\sin ^{2} \lambda\end{array}$ & $\begin{array}{l}k_{0}+1 \\
k_{0}+2\end{array}$ & $\begin{array}{l}\cos \lambda \cos \theta \\
\cos \lambda \sin \theta\end{array}$ & $\begin{array}{l}k_{1}+1 \\
k_{1}+2\end{array}$ & $\begin{array}{l}\cos ^{2} \lambda \cos 2 \theta \\
\cos ^{2} \lambda \sin 2 \theta\end{array}$ \\
\hline$k_{0}$ & $\sin q_{0} \lambda$ & $\begin{array}{l}k_{0}+3 \\
k_{0}+4\end{array}$ & $\begin{array}{l}\sin \lambda \cos \lambda \cos \theta \\
\sin \lambda \cos \lambda \sin \theta\end{array}$ & $\begin{array}{l}k_{1}+3 \\
k_{1}+4\end{array}$ & $\begin{array}{l}\sin \lambda \cos ^{2} \lambda \cos 2 \theta \\
\sin \lambda \cos ^{2} \lambda \sin 2 \theta\end{array}$ \\
\hline & & $\begin{array}{l}k_{1}-1 \\
k_{1}\end{array}$ & $\begin{array}{l}\sin q_{1} \lambda \cos \lambda \cos \theta \\
\sin q_{1} \lambda \cos \lambda \sin \theta\end{array}$ & $\begin{array}{l}\mathrm{K}-1 \\
\mathrm{~K}\end{array}$ & $\begin{array}{l}\sin q_{2} \lambda \cos ^{2} \lambda \cos 2 \theta \\
\sin q_{2} \lambda \cos ^{2} \lambda \sin 2 \theta\end{array}$ \\
\hline
\end{tabular}
The blanks in table 2 indicate zero coefficients.

2 The systematic and well-defined geographic variations of these Fourier coefficients are illustrated by an atlas of graphs for monthly median $f_{0} F_{2}$ for four seasonal months and for high and low years of solar activity [Jones, 1962].
TABLE 1. Geographic functions $\mathrm{G}_{k}(\lambda, \theta)$

Before considering methods for applying numerical maps in section 3 , we insert here a brief word of caution. The functions $G_{k}(\lambda, \theta)$ (in table 1 ) are not orthonormal relative to the coordinates $\left(\lambda_{i}, \theta_{i}\right)$ of the ionospheric stations; hence one cannot obtain a best representation (in the sense of least squares) of lower degree by merely truncating the series in (6). ${ }^{3}$ When a numerical map is originally produced, $a_{j}(\lambda, \theta)$ and $b_{j}(\lambda, \theta)$ are represented in terms of orthonormal functions, corresponding to the set of stations available for the particular month. Such series are then truncated at optimum places determined by the noise and are subsequently expressed in the form of (6) for convenience and simplicity in practical applications [Jones and Gallet, 1962, ch. 2].

\section{Methods for Applying Numerical Maps}

For applying numerical maps, a number of useful methods and techniques have been developed which eliminate slow and tedious hand work as much as possible and which make efficient use of automatic computing and plotting equipment. The discussion of such methods given in the present section is not intended to be exhaustive in nature but rather a starting place for developing further methods for applying numerical maps. Included are such topics as (1) efficient methods for evaluating $\Gamma(\lambda, \theta, t),(2)$ a general procedure for computing contour maps, and (3) a numerical check on the coefficients $D_{s k}$. The methods described below have been programed for use on several large-scale digital computers, the IBM 704 and 7090 and the CDC 1604.

\subsection{Evaluation of $\Gamma(\lambda, \theta, t)$}

Using the coefficients in table 2 , one can compute the value of $f_{0} F_{2}$ monthly median for September 1958 at any location on the earth and for any desired instant of time. This is the basic operation in most applications of numerical maps. The evaluation of $\Gamma(\lambda, \theta, t)$ for fixed values of $\lambda, \theta$, and $t$ can be performed in the straightforward manner indicated by (8),

3 the $G_{k}(\lambda, \theta)$ in table 1 are used, since they are the simplest functions which are linear combinations of the classical surface spherical harmonies [Byerly, 1893]. 
TABLE 2

TIME VARIATION

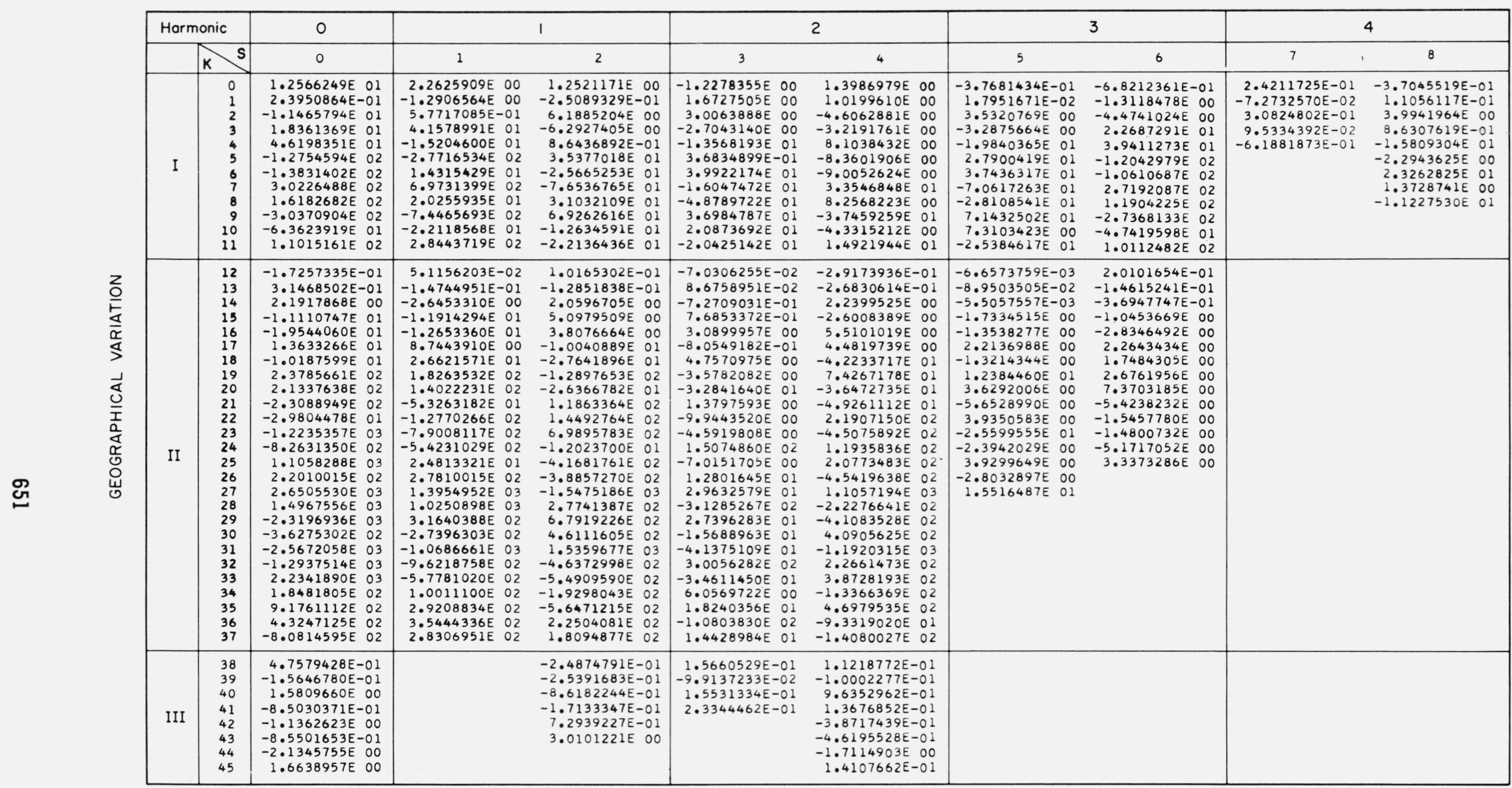

\begin{tabular}{|c|c|c|c|c|c|c|c|c|c|}
\hline \multirow{3}{*}{ 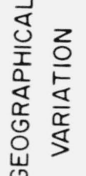 } & Harmonic & \multicolumn{2}{|c|}{5} & \multicolumn{2}{|r|}{6} & \multicolumn{2}{|r|}{7} & \multicolumn{2}{|c|}{8} \\
\hline & K 5 & 9 & 10 & 11 & 12 & 13 & 14 & 15 & 16 \\
\hline & $\begin{array}{l}0 \\
1 \\
2\end{array}$ & $\begin{array}{r}1.5436883 E-01 \\
-2.5472259 E-02 \\
-1.7009369 E-01\end{array}$ & $\begin{array}{r}2.5134453 E-01 \\
7.6121460 E-03 \\
-2.4425167 E-01\end{array}$ & $\begin{array}{r}-1.6932602 E-01 \\
-7.7820108 E-03 \\
1.8239843 E-01\end{array}$ & $\begin{array}{r}6.1690475 E-02 \\
-1.5589729 E-02 \\
-8.3470014 E-02\end{array}$ & $\begin{array}{r}-6.5109502 E-02 \\
2.1036162 E-03 \\
7.5380495 E-02\end{array}$ & $\begin{array}{r}-1.2627347 E-01 \\
-2.3577334 E-03 \\
1.4439629 E-01\end{array}$ & $\begin{array}{r}9.1845421 E-02 \\
-6.4546649 E-03 \\
-1.0264139 E-01\end{array}$ & $\begin{array}{r}-4.5376377 E-02 \\
4.9245555 E-04 \\
8.6725935 E-02\end{array}$ \\
\hline
\end{tabular}

I - Main latitudinal variation. Mixed latitudinal and longitudinal variation II - First order in longitude, III - Second order in longitude Notation: For each entry the number given by the first eight digits and sign is multiplied by the power of ten defined by the last two digits and sign. COEFFICIENTS $D_{S K}$ DEFINING THE FUNCTION $\Gamma(\lambda, \theta, \dagger)$ FOR MONTHLY MEDIAN fo $F 2$ (Mc/s) 
provided the coefficients $D_{s k}$ in table 2 are given. ${ }^{4}$ However, it is frequently required to compute the value of $\Gamma(\lambda, \theta, t)$ (a) at many locations for a fixed instant of time, or (b) at a fixed location for several different instants of time. In such cases considerable savings in computer cost can be achieved by using one of the following sets of procedures:

(a) Problem:

Given a fixed value $t_{0}$ of the local mean hour angle.

Compute $\Gamma\left(\lambda, \theta, t_{0}\right)$ for several points $(\lambda, \theta)$.

Procedure:

Step 1. Evaluate $\cos j t_{0}$ and $\sin j t_{0} j=1,2$, . .,$H$

Step 2. Compute:

$D_{k}\left(t_{0}\right)=D_{0, k}+\sum_{j=1}^{H}\left[D_{2 j, k} \cos j t_{0}+D_{2 j-1, k} \sin j t_{0}\right]$

for each $k=0,1, \ldots, K$ using step 1 and the coefficients $D_{s k}$.

Step 3. Evaluate $G_{k}(\lambda, \theta), k=0,1, \ldots, K$ for one of the given points $(\lambda, \theta)$.

$$
\text { Step 4. Compute } \Gamma\left(\lambda, \theta, t_{0}\right)=\sum_{k=0}^{K} D_{k}\left(t_{0}\right) G_{k}(\lambda, \theta) \text {. }
$$

Step 5. Repeat steps 3 and 4 for each remaining point $(\lambda, \theta)$.

Remark: The economy of this technique lies in the fact that steps 1 and 2 do not have to be repeated so long as $t_{0}$ remains ifixed.

(b) Problem:

Given fixed values of longitude $\theta_{0}$ and universal hour angle $T_{0}$.

Compute $\Gamma\left(\lambda, \theta_{0}, t_{0}\right)$, where $t_{0}=\theta_{0}+T_{0}$, for several values of $\lambda$.

Procedure:

Step 1. Follow steps 1 through 5 in (a) above, letting $t_{0}=\theta_{0}+T_{0}$ and giving $\theta$ the fixed value $\theta_{0}$ throughout. The points $(\lambda, \theta)$ in step 3 become $\left(\lambda, \theta_{0}\right)$.

(c) Problem: $\theta_{0}$.

Given fixed values of latitude $\lambda_{0}$ and longitude

Compute $\Gamma\left(\lambda_{0}, \theta_{0}, t\right)$ for several values of the local mean hour angle $t$.

Procedure:

Step 1. Evaluate $G_{k}\left(\lambda_{0}, \theta_{0}\right)$,

Step 2. Compute:

$$
k=0,1, \ldots, K \text {. }
$$

$a_{j}\left(\lambda_{0}, \theta_{0}\right)=\sum_{k=0}^{K} D_{2 j, k} G_{k}\left(\lambda_{0}, \theta_{0}\right), \quad j=0,1, \ldots, H$,

and

$b_{j}\left(\lambda_{0}, \theta_{0}\right)=\sum_{k=0}^{K} D_{2 j-1, k} G_{k}\left(\lambda_{0}, \theta_{0}\right)$,

$$
j=1,2, \ldots, H \text {. }
$$

${ }^{4}$ As was pointed out in section 2, the parameters $H=8, k_{0}=11, k_{1}=37, k_{2}=K=45$, $q_{0}=11, q_{1}=12$, and $q_{2}=3$ can be determined from table 2 .
Step 3. Evaluate sin $j t$ for $j=1,2, \ldots, H$, and $\cos j t$ for $j=1,2, \ldots, \mathrm{H}$

for one of the given values of $t$.

Step 4. Compute:

$$
\begin{aligned}
\Gamma\left(\lambda_{0}, \theta_{0}, t\right)=a_{0}\left(\lambda_{0}, \theta_{0}\right)+\sum_{j=1}^{H}\left[a_{j}\left(\lambda_{0}, \theta_{0}\right) \cos j t\right. \\
\left.+b_{j}\left(\lambda_{0}, \theta_{0}\right) \sin j t\right] .
\end{aligned}
$$

Step 5. Repeat steps 3 and 4 for each required value of $t$.

Remark: The economy of this technique lies in the fact that steps 1 and 2 are not repeated so long as the point $\left(\lambda_{0}, \theta_{0}\right)$ remains fixed.

\subsection{General Method for Computing Contour Maps}

In section 4 several types of contour maps of monthly median $f_{0} F_{2}$ are illustrated: (a) world and polar maps in universal time, (2) world maps in local mean time, and (3) maps in latitude and local mean time for fixed longitudes. Each of these maps has been computed using the coefficients $D_{s k}$ in table 2 and the general procedure described here. The procedure is of interest not only from the viewpoint of the applications illustrated in the present paper, but also since it can be applied to a wide variety of problems in which physical or other variations have been mapped in a numerical form.

The basic problem is the following: to locate (for a given time $t$ ) all points along certain meridian lines at which the function $\Gamma(\lambda, \theta, t)$ assumes any one of a given set of values $I_{0}, I_{1}, \ldots, I_{Q}$. For simplicity we assume the values $I_{q}$ to be equally spaced and increasing and hence defined by

$$
\begin{aligned}
& I_{q}=I_{0}+q\left(\frac{I_{Q}-I_{0}}{Q}\right) \quad q=0,1, \ldots, Q . \\
& I_{0}<I_{q} .
\end{aligned}
$$

A solution to this basic problem is given in the appendix. By repeated application of this solution one can compute any of the three types of contour maps mentioned above. The procedures are as follows.

\section{a. World Maps in Universal Time}

Let $T_{0}$ denote a given universal hour angle (sec. 2) and let

$$
\theta_{m}=360^{\circ}\left(\frac{m}{M}\right) \quad m=0,1, \ldots, M,
$$

and

$$
t_{m}=T_{0}+\theta_{m} \quad m=0,1, \ldots, M .
$$

Then for each pair $\left(\theta_{m}, t_{m}\right) \quad m=0,1, \ldots, M$ we compute all values of $\lambda$ satisfying.

$\Gamma\left(\lambda, \theta_{m}, t_{m}\right)=I_{q} \quad$ for some $q=0,1, \ldots, Q$,

using the method given in the appendix. By 
choosing $M$ sufficiently large, the points on the map can be brought closely enough together to define clearly each contour. In practice $M$ can be made as large as we please, but for purposes of economy it is desirable to make it as small as can be allowed. For most ionospheric characteristics, $M=24$ is large enough.

\section{b. World Maps in Local Mean Time}

Let $t_{0}$ denote a given local mean hour angle (sec. 2) and let $\theta_{m}$ be given by (10). Using the method in the appendix, we compute (for each $\theta_{m}$ ) all values of $\lambda$ satisfying

$$
\Gamma\left(\lambda, \dot{\theta}_{m}, t_{0}\right)=I_{q} \quad \text { for some } q=0,1, \ldots, Q .
$$

Again the number $M$ is chosen as described above.

\section{c. Maps in Latitude and LMT for Fixed $\theta$}

Let $\theta_{0}$ denote a given longitude and let

$$
t_{m}=180^{\circ}[2 m / M-1], \quad m=0,1, \ldots, M
$$

denote a set of local mean hour angles. Again using the solution given in the appendix, we can locate all values of $\lambda$ such that

$$
\Gamma\left(\lambda, \theta_{0}, t_{m}\right)=I_{q} \quad \text { for some } \quad q=0,1, \ldots, Q
$$

for each value of $m$. As before, we must choose $M$ large enough so that the points on the map clearly define each contour. $M=24$ is sufficient for most ionospheric characteristics.

Before considering the graphical illustrations in section 4 computed by the above procedures, we describe briefly a useful numerical check which can be made on the coefficients $D_{s k}$ before each application.

\subsection{A Numerical Check on the Coefficients $\mathrm{D}_{s k}$}

In the normal operations of handling, sorting, and reproducing decks of punched cards and converting to magnetic tapes and reading into a computer, there is always the possibility that a card will be misplaced or that a number will be transferred or reproduced incorrectly. For this reason it is recommended that certain periodic checks be made when applying the coefficients $D_{s k}$ defining a numerical map.

One such test that can be performed automatically by the computer is the following. Given a set of correct values of the function $\Gamma(\lambda, \theta, t)$ for a few values of the independent variables $\lambda, \theta$, and $t$, one can test all of coefficients $D_{s k}$ by recomputing the given values of $\Gamma(\lambda, \theta, t)$. If the functional values agree (to, say, six digits) then the coefficients can be used with confidence. As an example we give a set of functional values in table 3 that can be used to test the coefficients in table 2 .
TABLE 3. Functional values of $\Gamma(\lambda, \theta, \mathrm{t})$ for testing coefficients

\begin{tabular}{|c|c|c|}
\hline LMT & $t$ & $\boldsymbol{\Gamma}(\lambda, \theta, t)$ \\
\hline $\begin{array}{l}00 \\
01 \\
02 \\
03 \\
04\end{array}$ & $\begin{array}{l}-180^{\circ} \\
-165^{\circ} \\
-150^{\circ} \\
-135^{\circ} \\
-120^{\circ}\end{array}$ & $\begin{array}{c}\text { Mc/s } \\
6.8834498 \\
6.6014013 \\
6.2867521 \\
6.0181661 \\
5.7592264\end{array}$ \\
\hline $\begin{array}{l}05 \\
06 \\
07 \\
08 \\
09\end{array}$ & $\begin{array}{l}-105^{\circ} \\
-90^{\circ} \\
=75^{\circ} \\
-60^{\circ} \\
-45^{\circ}\end{array}$ & $\begin{array}{r}6.0961668 \\
7.6478070 \\
9.6392061 \\
10.9863478 \\
11.7429891\end{array}$ \\
\hline $\begin{array}{l}10 \\
11 \\
12 \\
13 \\
14\end{array}$ & $\begin{array}{r}-30^{\circ} \\
-15^{\circ} \\
0^{\circ} \\
15^{\circ} \\
30^{\circ}\end{array}$ & $\begin{array}{l}\text { 12. } 2211317 \\
\text { 12. } 3494360 \\
12.1784082 \\
11.8577095 \\
11.4744272\end{array}$ \\
\hline $\begin{array}{l}15 \\
16 \\
17 \\
18 \\
19\end{array}$ & $\begin{array}{r}45^{\circ} \\
60^{\circ} \\
75^{\circ} \\
90^{\circ} \\
105^{\circ}\end{array}$ & $\begin{array}{r}11.1244725 \\
10.8307340 \\
10.5763241 \\
10.2428354 \\
9.6362065\end{array}$ \\
\hline $\begin{array}{l}20 \\
21 \\
22 \\
23\end{array}$ & $\begin{array}{l}120^{\circ} \\
135^{\circ} \\
150^{\circ} \\
165^{\circ}\end{array}$ & $\begin{array}{l}\text { 8. } 8870622 \\
\text { 8. } 2365329 \\
7.6430851 \\
7.1626558\end{array}$ \\
\hline
\end{tabular}
$\mathrm{D}_{\mathrm{sk}}$ for monthly median $\mathrm{f}_{0} \mathrm{~F}_{2}(M c / s)$, September 1958 (see table 2)

\section{Graphical Representations From Numerical Maps}

As was mentioned above, many interesting and useful results can be produced from a numerical map. The basic application, using the coefficients $D_{s k}$, is to compute the value of $\Gamma(\lambda, \theta, t)$-i.e., of the ionospheric characteristic - at any desired location and instant of time in the three-dimensional space of latitude, longitude, and local mean time. ${ }^{5}$ For the purpose of illustration, a schematic diagram of this space is given in figure 1. In this diagram several types of (two-dimensional) cuts and (onedimensional) lines are shown, on each of which a graphical representation of the ionospheric characteristic can be produced from its numerical map. These cuts and lines, summarized in table 4, should be considered only a suggestive sample and not an exhaustive set.

TABLE 4. Summary of cuts and lines in the three-dimensional

\begin{tabular}{|c|c|c|c|c|}
\hline & Latitude & Longitude & LMT & UT \\
\hline $\begin{array}{l}\text { Surfaces: } \\
\text { Cut I } \\
\text { Cut II } \\
\text { Cut III }\end{array}$ & $\begin{array}{l}\mathrm{V} \\
\mathrm{V} \\
\mathrm{V}\end{array}$ & $\begin{array}{l}\mathrm{V} \\
\mathrm{F} \\
\mathrm{V}\end{array}$ & $\begin{array}{l}\mathrm{F} \\
\mathrm{V}\end{array}$ & F \\
\hline $\begin{array}{l}\text { Lines: } \\
\text { Line A } \\
\text { Line B } \\
\text { Line C } \\
\text { Line D }\end{array}$ & $\begin{array}{l}\mathrm{F} \\
\mathrm{F} \\
\mathrm{V} \\
\mathrm{F}\end{array}$ & $\begin{array}{l}\mathrm{F} \\
\mathrm{V} \\
\mathrm{F} \\
\mathrm{V}\end{array}$ & $\begin{array}{l}\mathrm{V} \\
\mathrm{F} \\
\mathrm{F}\end{array}$ & $\mathrm{F}$ \\
\hline
\end{tabular}
space of $\lambda, \theta, \mathrm{t}$

( $\mathrm{F}=$ fixed $\mathrm{V}=$ variable)

${ }^{5}$ Simple procedures for making this application were given in section 3.2 . These can be performed with such speed by an electronic computer that very extensive applications are practical. 


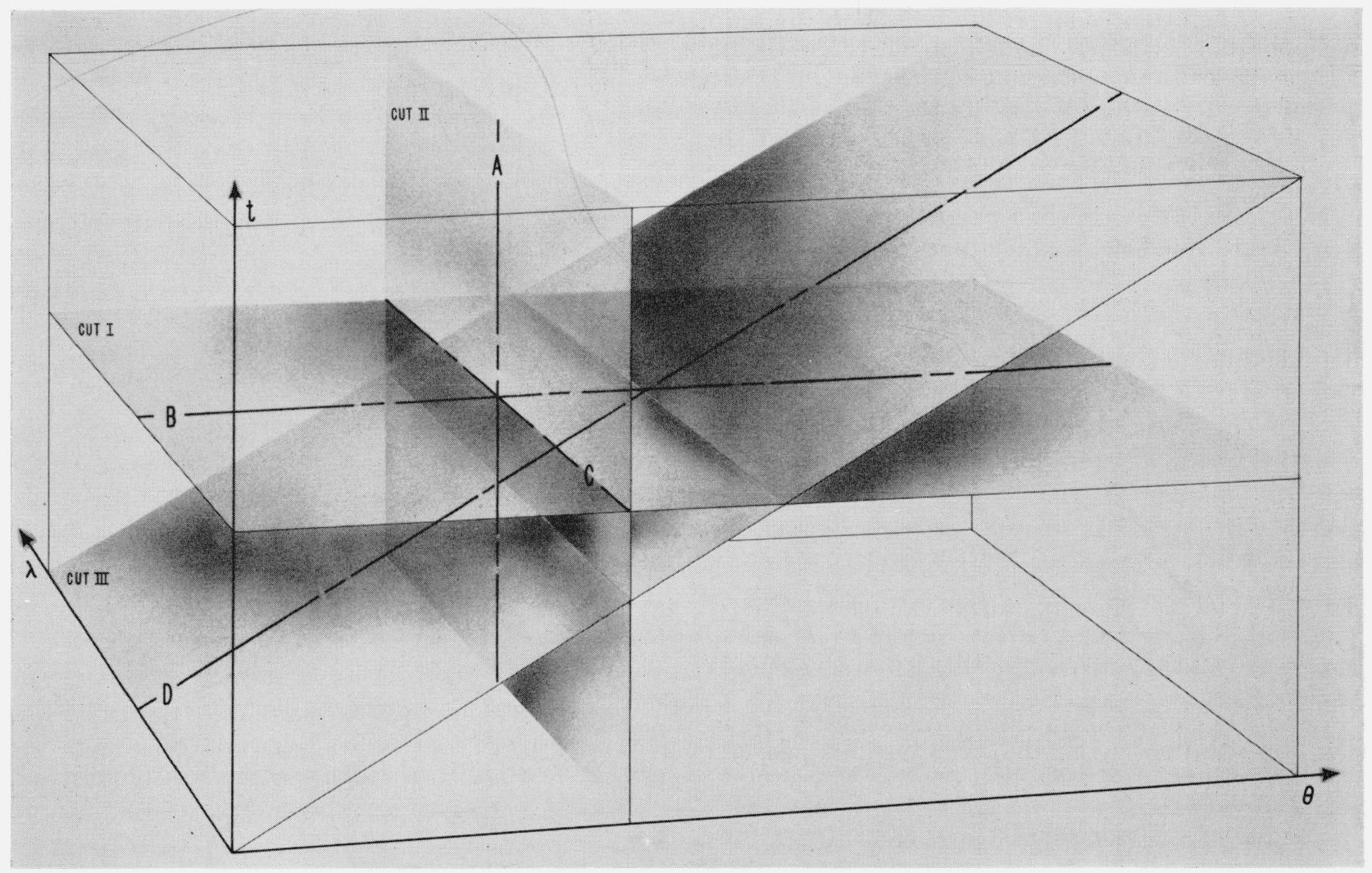

Figure 1. Three-dimensional space of $\lambda, \theta, t$.

$\lambda=$ geographic latitude, $\theta=$ geographic longitude,

$t=$ local mean hour angle,

$T=$ universal hour angle.

In this section, examples are given of graphical representations for each cut and line in table 4, such representations being computed directly from the coefficients in table 2. For each line in the threedimensional space (fig. 1), the graphical representation is a simple two-dimensional graph computed from $\Gamma(\lambda, \theta, t)$ in the obvious manner. For cuts, however, the representations take the form of contour maps on which selected values of $\Gamma(\lambda, \theta, t)$ are traced throughout a plane region. Such maps are computed by solving high degree polynomial equations in $\sin \lambda$ for points lying on selected meridian lines (sec. 3.2). Since $\Gamma(\lambda, \theta, t)$ is defined continuously throughout the space, points on the contours can be obtained as close together as desired. The following are illustrations of such graphs and contour maps.

\subsection{World Maps in Universal Time}

World maps of monthly median $f_{0} F_{2}$ in universal time (Cut III) are illustrated in figures 2 and 3. Such maps are probably the most useful for problems of radio propagation, since they represent the ionospheric characteristic all over the world at a fixed instant. The maps, given for the four hours, $\mathrm{U}^{\prime} \mathrm{T}=00$, $06,12,18$, are presented in such a way that it is always midnight in LMT at the extreme left boundary and noon in LMT at the center. This type of presentation enables one to see the relatively small continuous deformations in the ionosphere as the earth rotates about its axis.

Since the geographic poles are singular points in the numerical maps, the function $\Gamma(\lambda, \theta, t)$ is physically meaningless in the immediate neighborhood of the north and south poles. Therefore, in these regions dashed lines (interpolated by hand) have been inserted in the contour maps to give a meaningful extention to the poles. Similarly dashed lines have been inserted in figures $4,5,8$, and 9 .

\subsection{Polar Maps in Universal Time}

Polar maps of monthly median $f_{0} F_{2}$ in universal time (Cut III), illustrated in figures 4 and 5 , are similar to the world maps in UT (figs. 2 and 3 ). Such maps are more convenient for working with radio circuits passing over the polar caps than the (rectangular) world maps in U'T shown in figures 2 and 3.

\subsection{World Maps in Local Mean Time}

World maps of monthly median $f_{0} F_{2}$ in local mean time (Cut I) represent the worldwide geographic variations which are not produced by changes in the hour angle of the sun (ings. 6 and 7 ). Such maps are particularly useful in studying the complex and, 

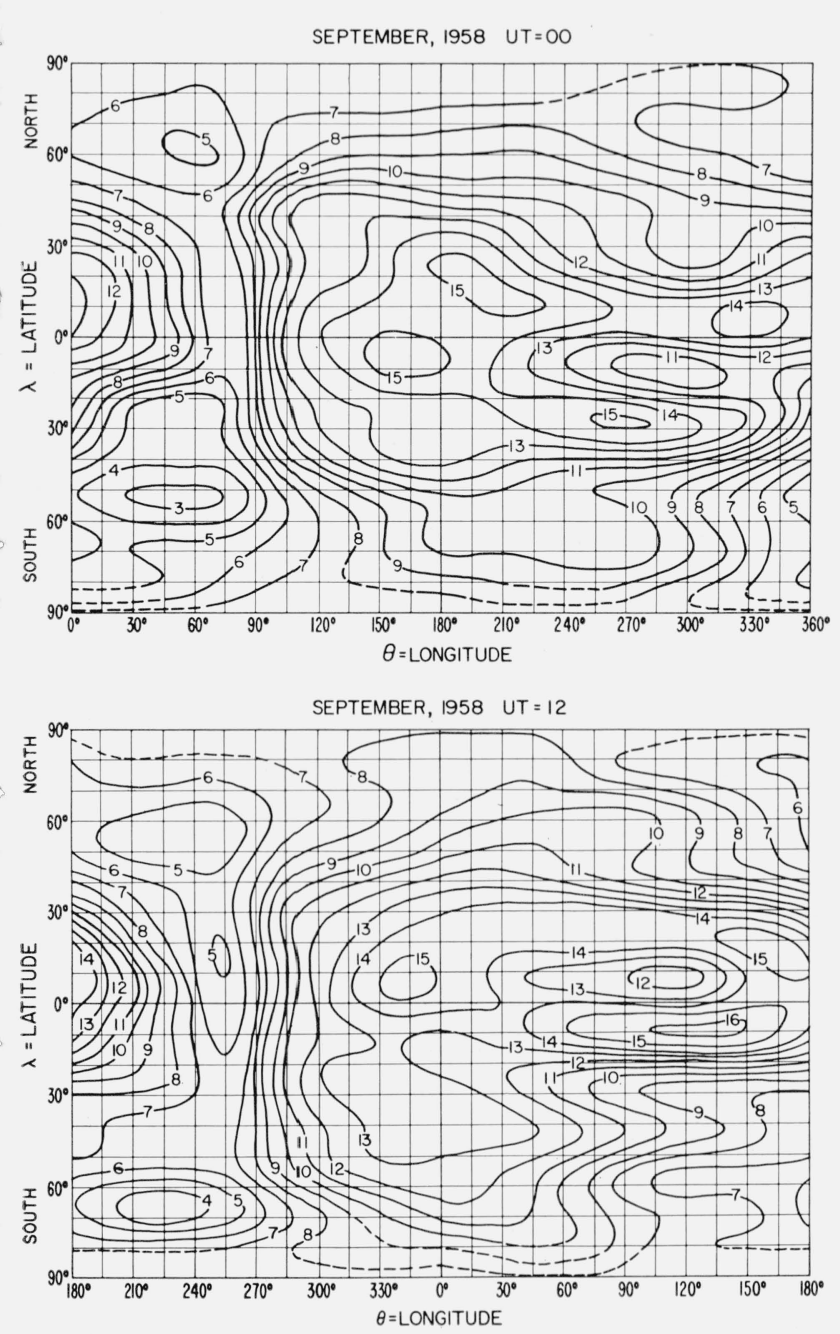

Figure 2. World maps of monthly median $\mathrm{f}_{0} \mathrm{~F}_{2}(M c / s)$ in universal time from the function $\Gamma(\lambda, \theta, t)$.

to some extent, irregular geographic variations due to seasonal changes, the earth's magnetic field, and other factors affecting the ionosphere. The maps are given for four different hours (LMT $=00,06,12$, 18), illustrating the different conditions occurring at midnight, sunrise, noon, and late afternoon, respectively.

\subsection{Maps in Latitude and LMT}

Maps of monthly median $f_{0} F_{2}$ in latitude and LMT (Cut II) are given in figures 8 and 9 for several fixed values of longitude. These maps are similar to the "longitude zone" maps presently given in the CRPL-D Series. However, in contrast with the $\mathrm{D}$ series, it is not assumed here that longitudinal variation of the ionospheric characteristic is constant within a given zone.
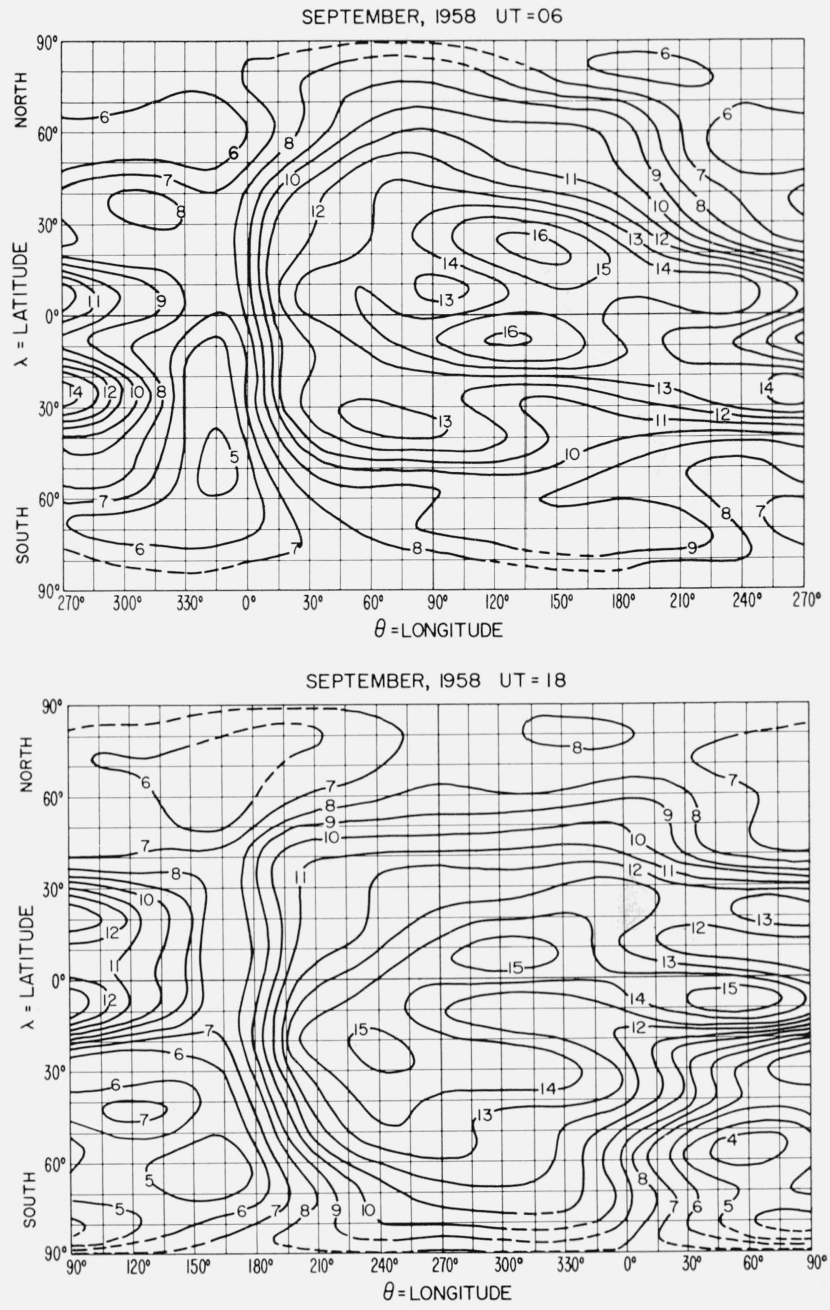

Figure 3. World maps of monthly median $\mathrm{f}_{0} \mathrm{~F}_{2}(M c / s)$ in universal time from the function $\Gamma(\lambda, \theta, t)$.

\subsection{Diurnal Variation}

The diurnal variation for monthly median $f_{0} F_{2}$ (Line A) is illustrated in figures 10 and 11 for several different locations on the earth (the points of intersection of the two meridians $90^{\circ} \mathrm{E}$ and $285^{\circ} \mathrm{E}$ with the five parallels $0^{\circ}, \pm 20^{\circ}, \pm 50^{\circ}$ ). These graphs illustrate the strong changes in the diurnal variation with geographic position.

\subsection{Longitudinal Variation (Fixed LMT)}

The longitudinal variation of monthly median $f_{0} F_{2}$ (Line B) for a fixed instant of LMT is illustrated by the graphs in figure 12 . The changes in this variation (corresponding to different latitudes and hours of LMT) are typified by the graphs for each of the hours, 00 and 12 , and for each of the latitudes $0^{\circ}$, $\pm 20^{\circ}, \pm 50^{\circ}$. 

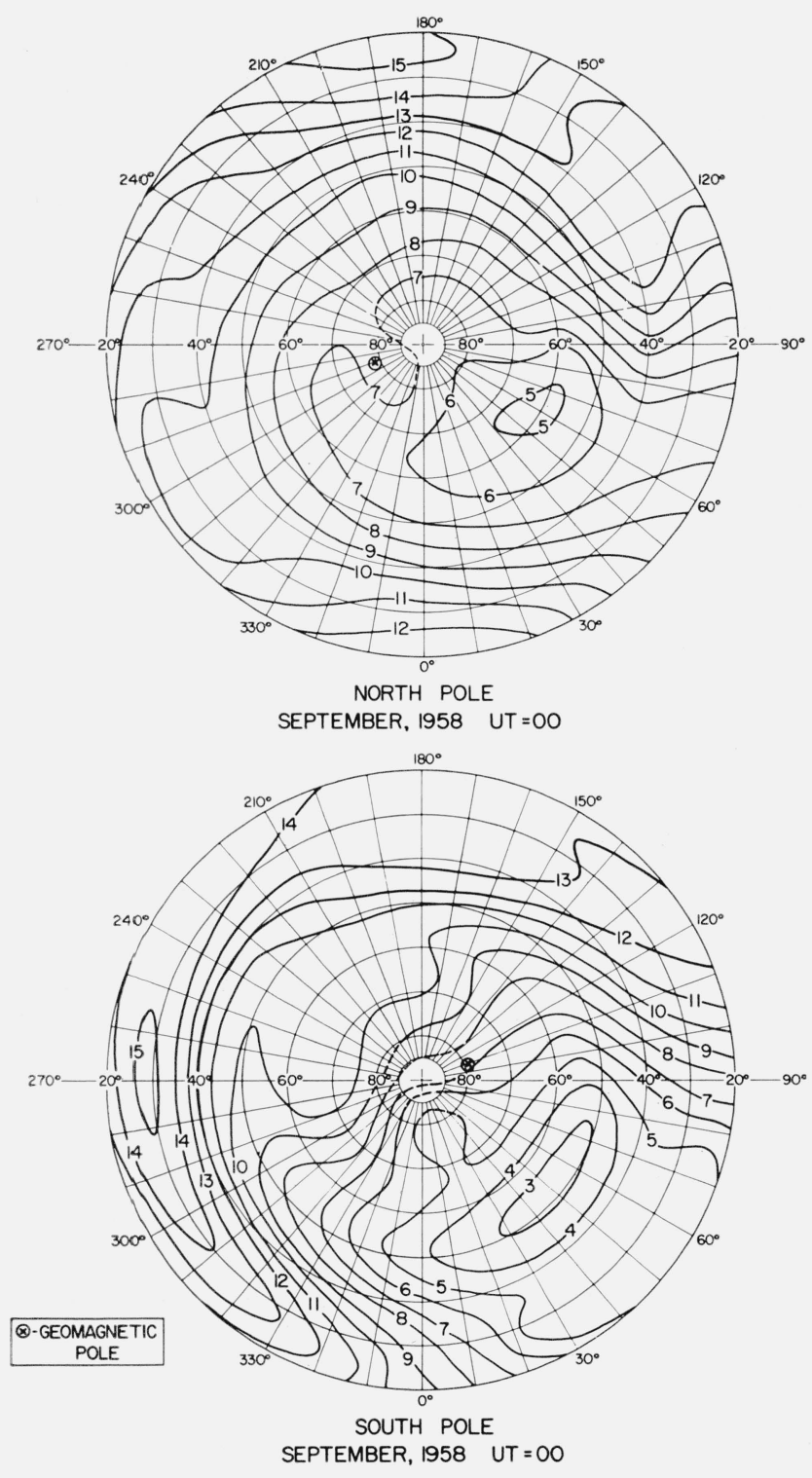

Figure 4. Polar maps of monthly median $\mathrm{f}_{0} \mathrm{~F}_{2}(M c / s)$ in universal time from the function $\Gamma(\lambda, \theta, t)$.

\subsection{Latitudinal Variation (Fixed LMT)}

The latitudinal variation of monthly median $f_{0} F_{2}$ (Line C) for a fixed instant of LM'T is shown by the graphs in figure 13. Graphs are given for each of the fixed longitudes $0^{\circ} \mathrm{E}, 90^{\circ} \mathrm{E}, 180^{\circ} \mathrm{E}$, and $270^{\circ} \mathrm{E}$, for each hour, LMT $=00$ and 12 .

\subsection{Longitudinal Variation (Fixed UT)}

The variation of monthly median $f_{0} F_{2}$ with longitude (Line D) for a fixed instant of U'T is illustrated by the graphs in figure 14, for the two hours, $\mathrm{U}^{\prime} \mathrm{T}=00$ and 12 , for each of the fixed latitudes $0^{\circ}, \pm 20^{\circ}, \pm 50^{\circ}$.

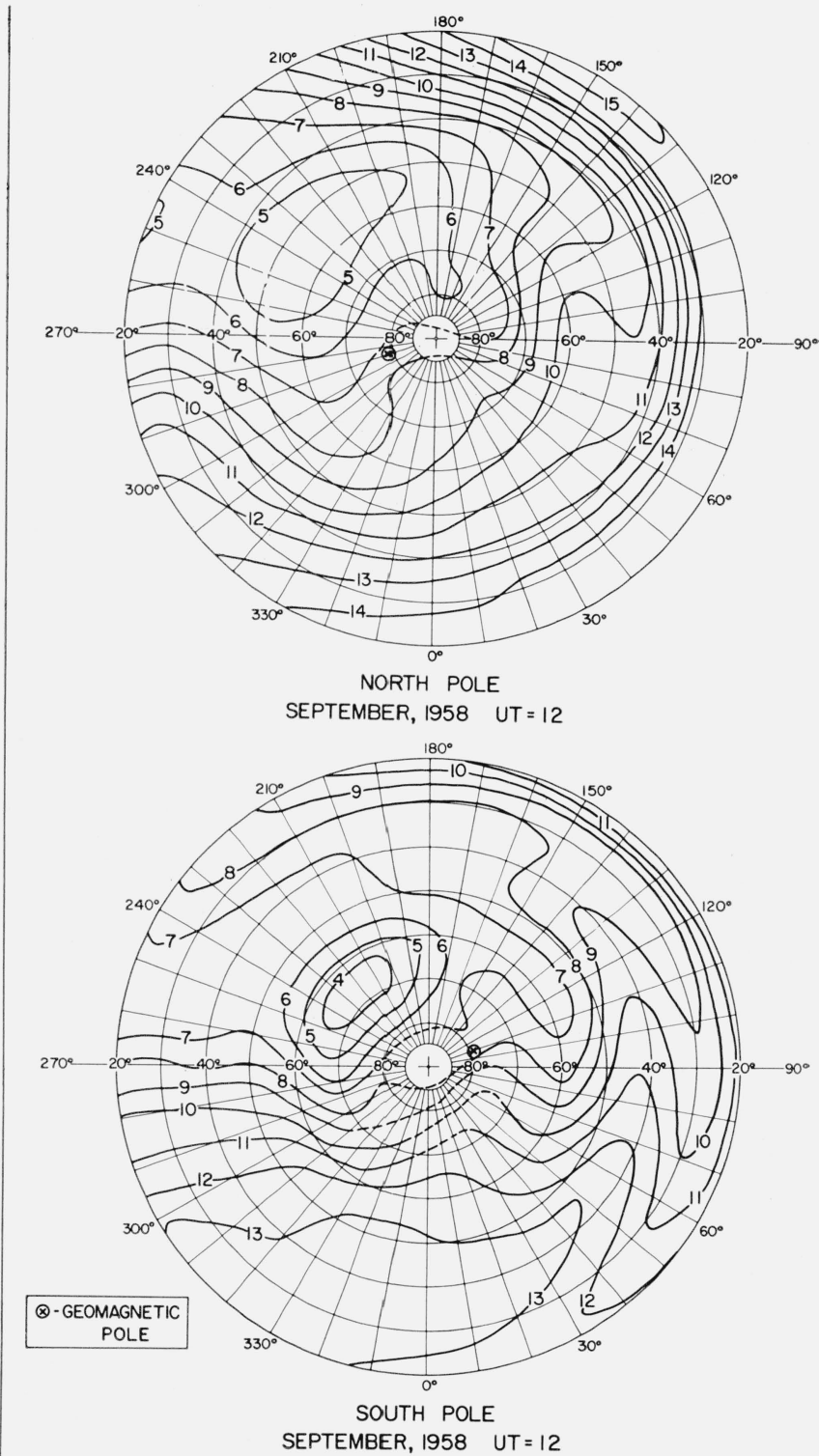

Figure 5. Polar maps of monthly median $\mathrm{f}_{0} \mathrm{~F}_{2}\left(M_{c} / s\right)$ in universal time from the function $\Gamma(\lambda, \theta, t)$.

\section{Summary}

It has been pointed out that a numerical map is defined by means of a relatively small table of numerical coefficients with which many useful applications can be made. In particular, one can compute the value of the ionospheric characteristic at any desired location and instant of time. By means of this operation a wide variety of graphical representations can be computed automatically and plotted by machine. These include, for example, world and polar contour maps in either universal time or local mean time, illustrations of which were given in section 4 . 
SEPTEMBER, 1958 LMT $=00$

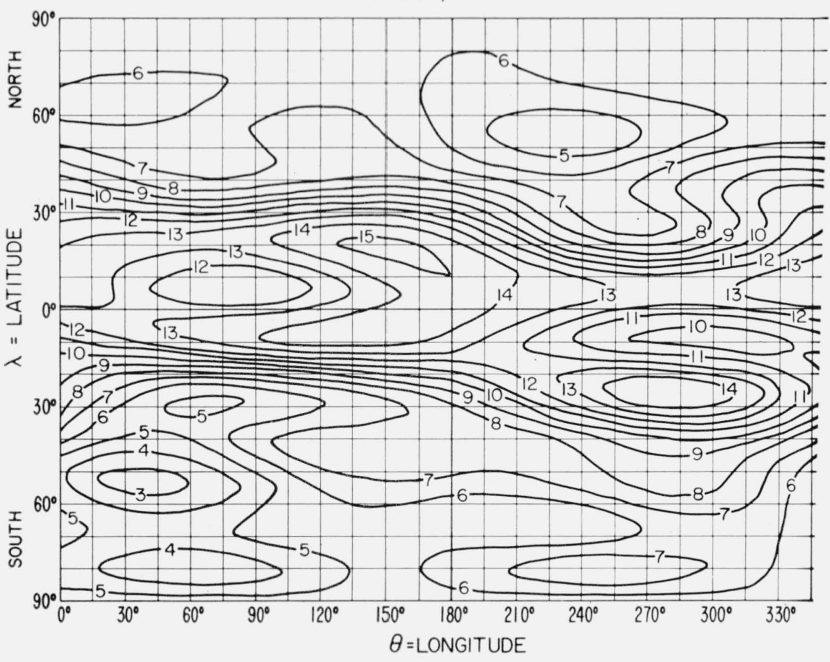

SEPTEMBER, 1958 LMT $=12$

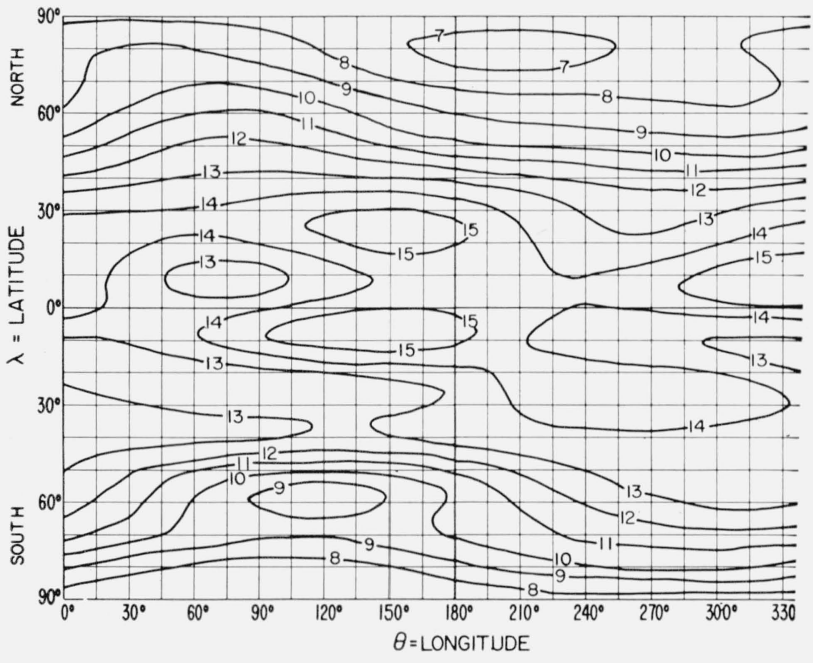

Figure 6. World maps of monthly median $\mathrm{f}_{0} \mathrm{~F}_{2}(M c / s)$ in local mean time from the function $\Gamma(\lambda, \theta, t)$.

Although such graphical displays have many important uses, it should be re-emphasized that numerical maps can be used to the greatest advantage and efficiency within the computer-that is, by letting the machine compute the values of the characteristic rather than by reading them manually from a contour map. The computer can do this much faster and more accurately than a person reading and interpolating between maps.

\section{Appendix: Basic Problem in Computing Contour Maps}

Let us assume that we have been given a table of coefficients $D_{s k}$ defining a numerical map $\Gamma(\lambda, \theta, t)$ for an ionospheric characteristic (for example, the coefficients in table 2 for monthly median $f_{0} F_{2}$,
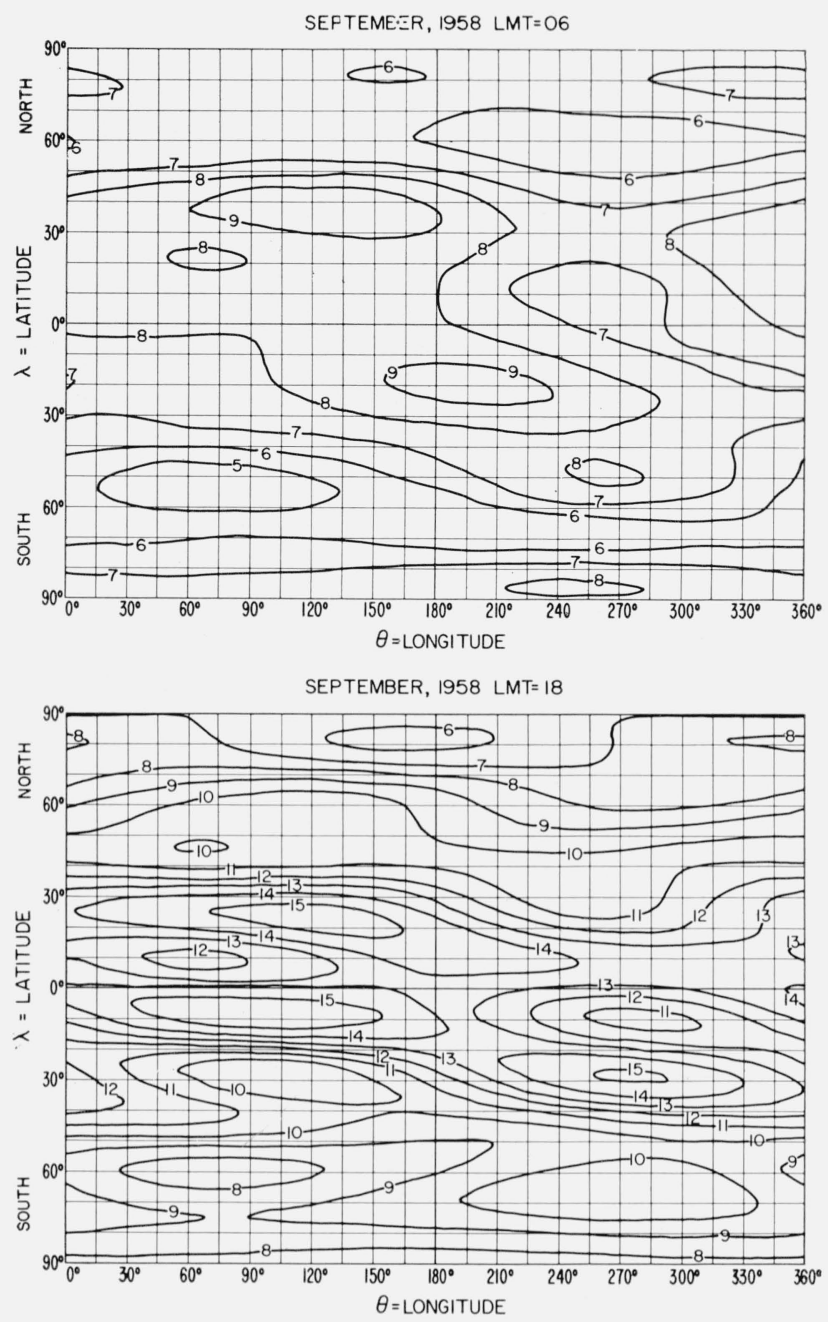

Figure 7. World maps of monthly median $\mathrm{f}_{0} \mathrm{~F}_{2}(M c / s)$ in local mean time from the function $\Gamma(\lambda, \theta, t)$.

September 1958), so that we can evaluate $\Gamma(\lambda, \theta, t)$ at any desired point in the three-dimensional space of $\lambda, \theta$, and $t$. Then the problem considered here is that of locating, for given fixed values of $\theta$ and $t$, all values of $\lambda$ for which

$$
\Gamma(\lambda, \theta, t)=I_{q} \quad \text { for some } q=0,1, \ldots, Q
$$

(see sec. 3.2). Thus the problem consists in solving high degree polynomial equations in $\sin \lambda$ for points $\lambda$ lying along selected meridians. The method we employ has two main steps, (1) to isolate the roots within certain small intervals and (2) to approach each root by a method of successive approximation. We begin the discussion with a description of two basic parameters which must be determined. These parameters are associated with the function $\Gamma(\lambda, \theta, t)$. 


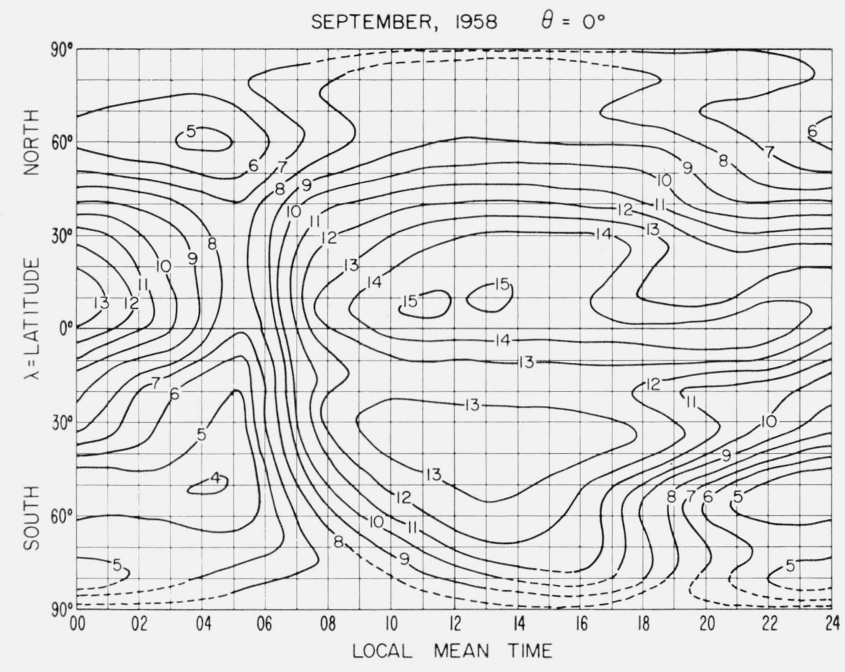

SEPTEMBER, $1958 \quad \theta=180^{\circ}$

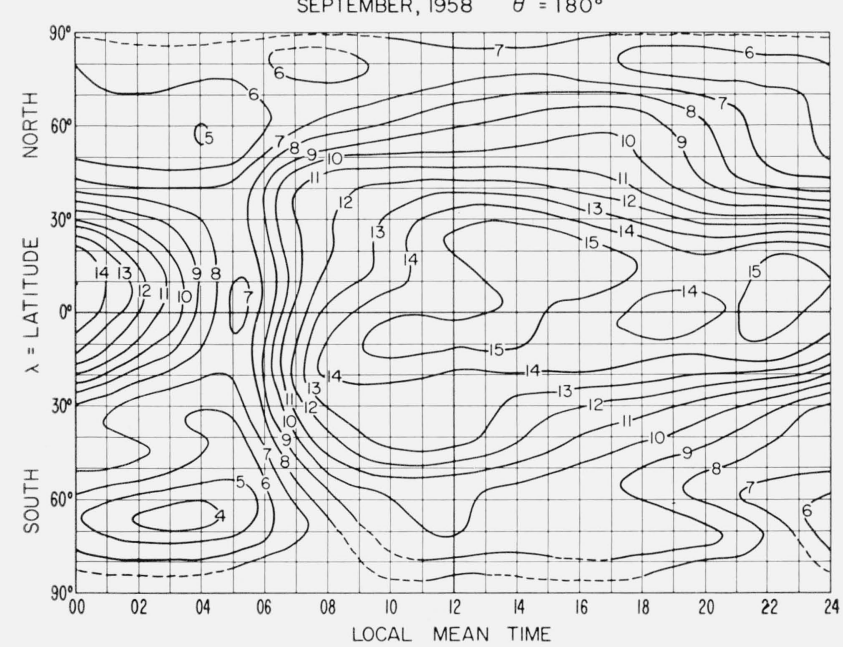

Figure 8. Maps of monthly median $\mathrm{f}_{0} \mathrm{~F}_{2}(\mathrm{Mc} / \mathrm{s})$ in latitude and $L M T$ at fixed longitudes from the function $\Gamma(\lambda, \theta, t)$.

\subsection{Parameters $\epsilon$ and $N$ Associated With $\Gamma(\lambda, \theta, t)$}

In order to solve the problem outlined above, two parameters $\epsilon$ and $N$ are needed. The determination of these parameters depends upon certain general properties of the function $\Gamma(\lambda, \theta, t)$ as follows. The positive number $\epsilon$ determines ${ }^{6}$ the permissible error in an approximation to a root of (16) in the sense that $\lambda$ will be considered a solution to (16) if

$$
\left|\Gamma(\lambda, \theta, t)-I_{q}\right| \leqq \epsilon \quad \text { for some } q=0,1, \ldots, Q \text {. }
$$

For a very flat part of the function $\Gamma(\lambda, \theta, t)$, large variations in the independent variables make only small changes in $\Gamma(\lambda, \theta, t)$. Thus, for a given $\epsilon$, the error in $\lambda$ is proportional to the "flatness" of $\Gamma(\lambda, \theta, t)$.

${ }^{6}$ As can be seen, $\epsilon$ is not itself the upper bound of the error in $\lambda$ but only determines what that upper bound will be for a particular function $\Gamma(\lambda, \theta, t)$.
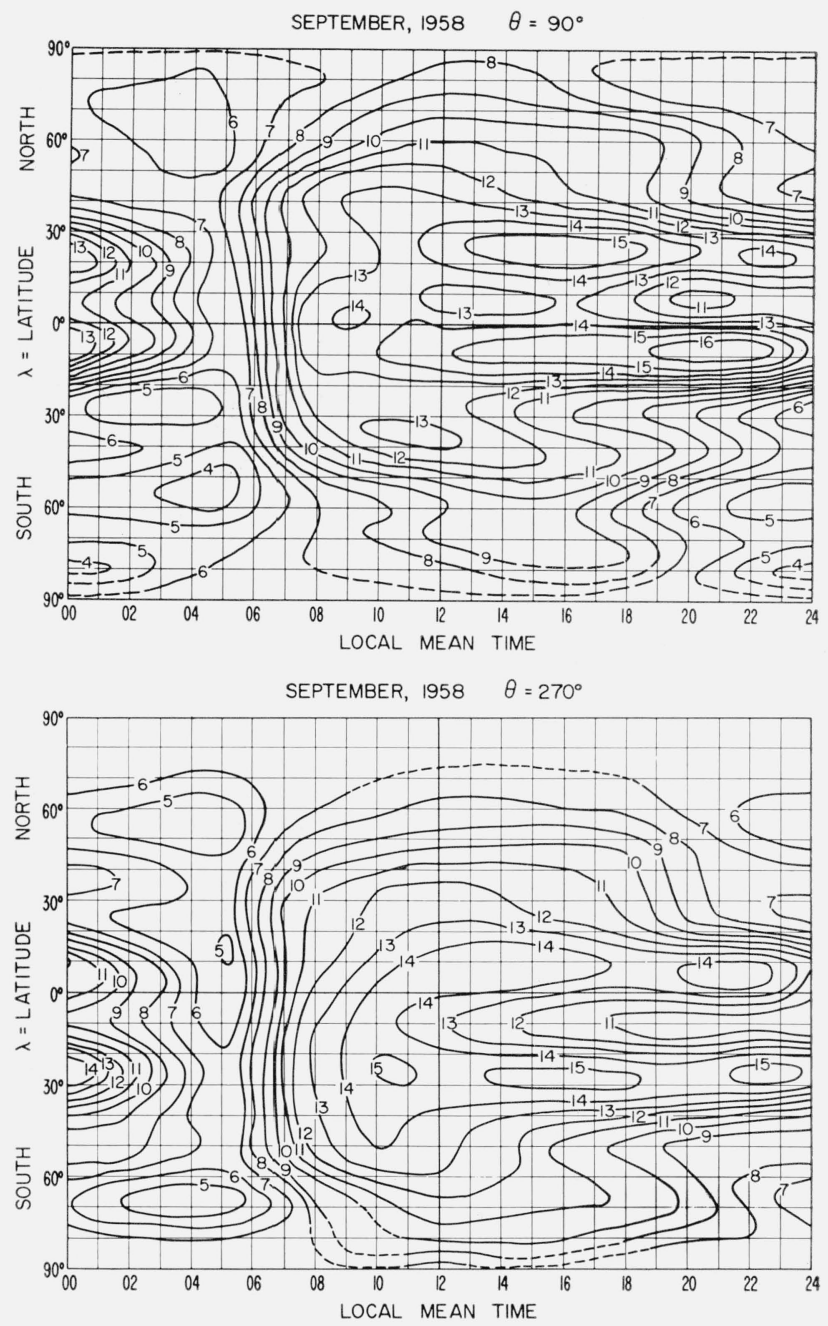

Figure 9. Maps of monthly median $\mathrm{f}_{0} \mathrm{~F}_{2}(\mathrm{Mc} / \mathrm{s})$ in latitude and $L M T$ at fixed longitudes from the function $\Gamma(\lambda, \theta, t)$.

Since the roots of (16) are obtained by successive approximations, it is desirable for economy to choose $\epsilon$ no larger than necessary. For most ionospheric characteristics a suitable choice is $\epsilon=0.0001$.

The number $N$ depends upon $\Gamma(\lambda, \theta, t)$ in somewhat the opposite sense. As will be seen, successive approximations to roots of (16) are to be made by means of linear interpolation within the intervals

$$
\lambda_{n-1}<\lambda<\lambda_{n} \quad n=1,2, \ldots, N
$$

where

$$
\lambda_{n}=\left[2\left(\frac{n}{N}\right)-1\right] 90^{\circ} \quad n=0,1, \ldots, N
$$

Therefore $N$ must be chosen large enough so that, for fixed values of $\theta$ and $t$, the function $\Gamma(\lambda, \theta, t)$ will be approximately linear within the intervals (18). If there are relative maxima or minima of $\Gamma(\lambda, \theta, t)$ 


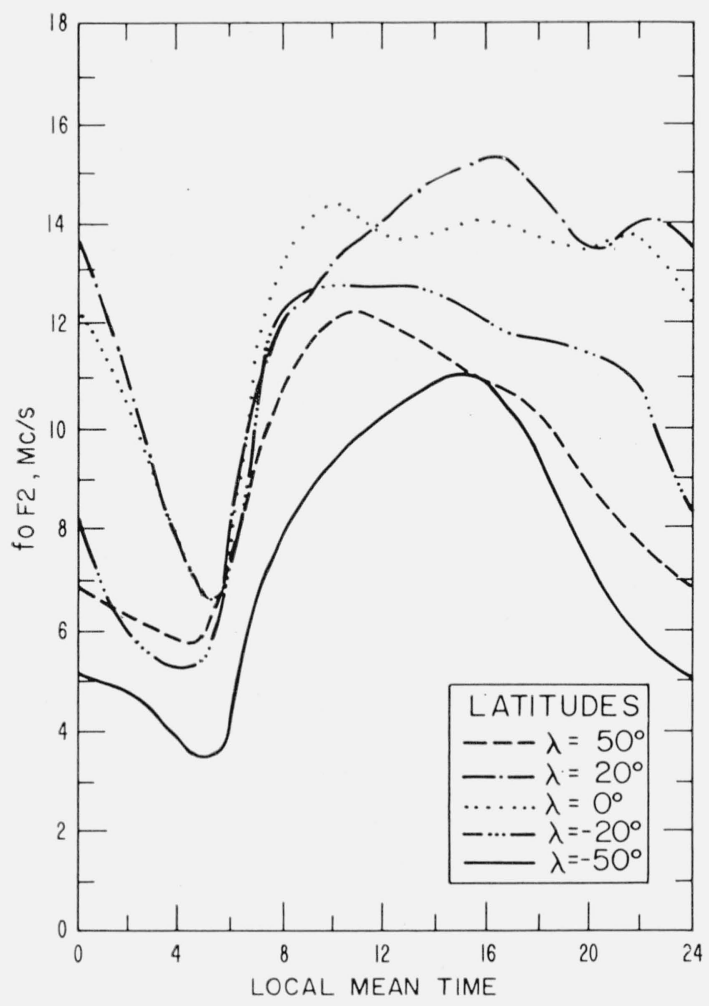

Figure 10. Diurnal variation of monthly median $\mathrm{f}_{0} \mathrm{~F}_{2}$ from the function $\Gamma(\lambda, \theta, t)$. September 1958 (Fixed longitude: $\left.\theta=90^{\circ}\right)$

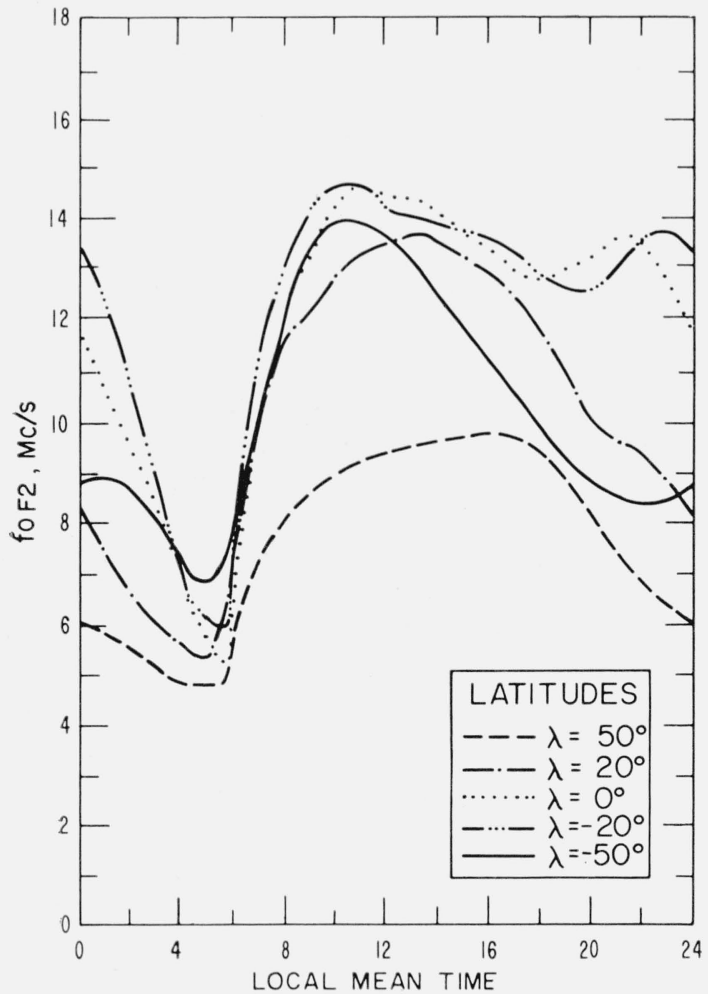

FiguRE 11. Diurnal variation of monthly median $\mathrm{f}_{0} \mathrm{~F}_{2}$ from the function $\Gamma(\lambda, \theta, t)$. September 1958 (Fixed longitude: $\left.\theta=285^{\circ}\right)$.

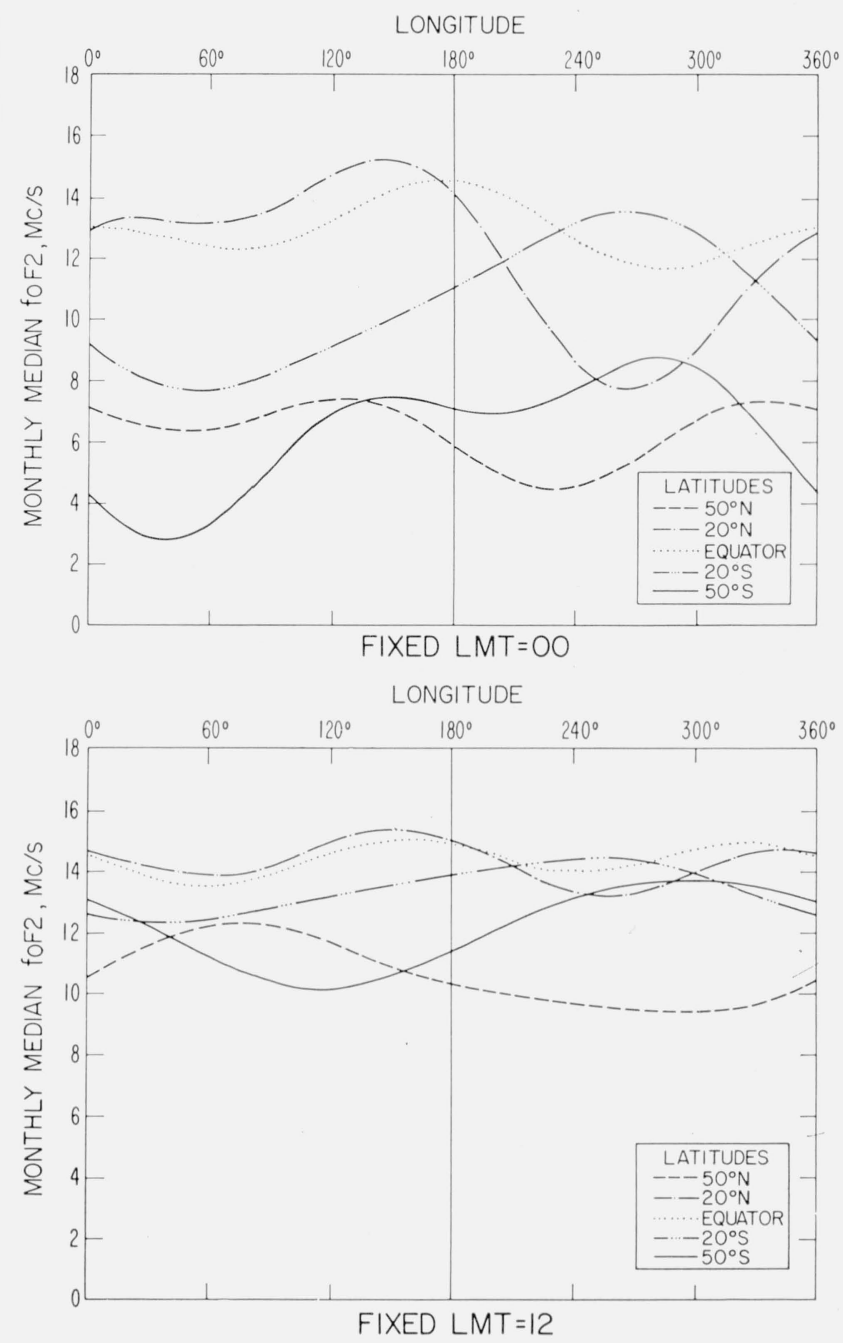

FIGURE 12. Longitudinal variation of monthly median $\mathrm{f}_{0} \mathrm{~F}_{2}$ from the function $\Gamma(\lambda, \theta, t)$ for September 1958.

within certain of the intervals (18), a few points on the map could be lost.

For the sake of economy one would like to choose $N$ no larger than necessary. For most ionospheric characteristics it has been found that $N=36$ is sufficient. A typical example of the variation of $\Gamma(\lambda, \theta, t)$ for monthly median $f_{0} F_{2}$, September 1958 , with $\theta=270^{\circ}$ and $t=-180^{\circ} \quad(L M T=00)$ is shown in figure 15 . The vertical lines spaced every $5^{\circ}$ illustrate the type of subdivision resulting from the choice of $N=36$.

In the remaining part of the appendix we shall assume that $\epsilon$ and $N$ have been appropriately chosen and that $\theta$ and $t$ are held fixed; hence for simplicity we shall write $\Gamma(\lambda)$ in place of $\Gamma(\lambda, \theta, t)$.

\subsection{Isolation of the Roots in the $n$th Interval}

We consider first the problem of determining the number of roots in the $n$th interval (18). We 

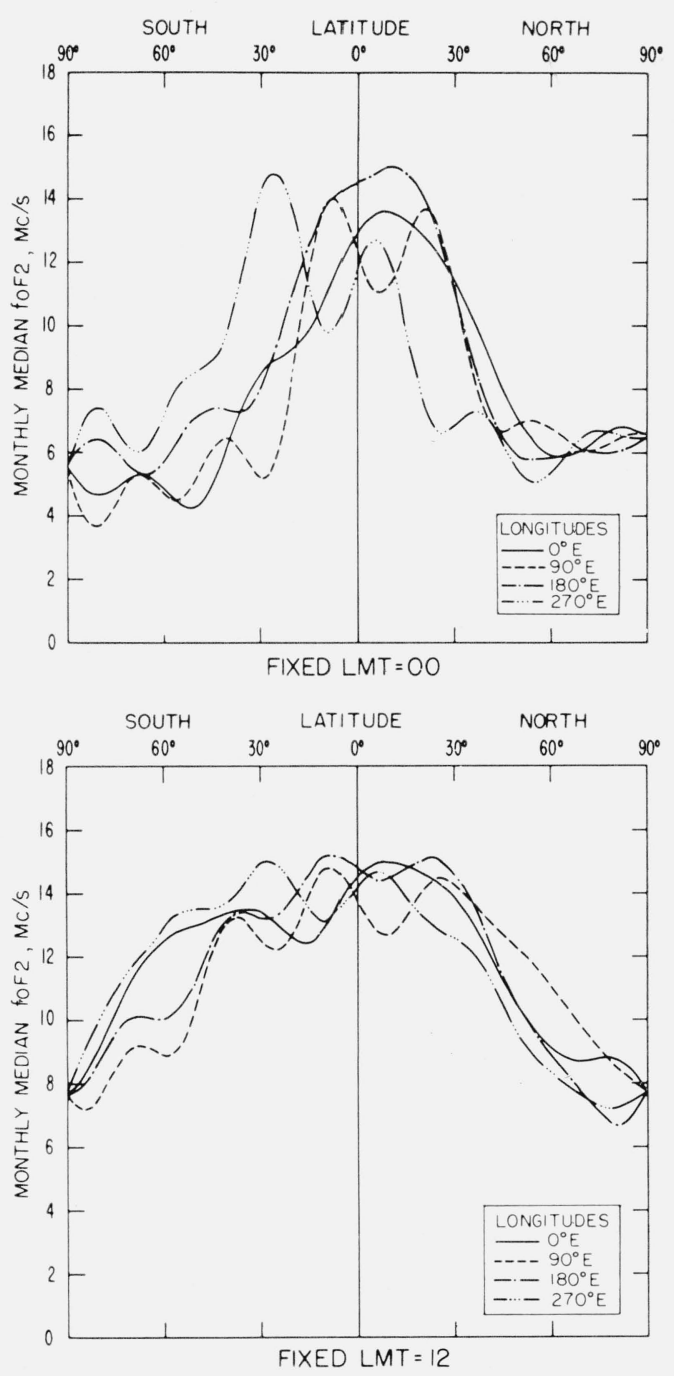

FIGURE 13. Latitudinal variation of monthly median $\mathrm{f}_{0} \mathrm{~F}_{2}$ from the function $\Gamma(\lambda, \theta, t)$ for September 1958.

define $\mu_{n}$ as that value of $q(q=0,1, \ldots, Q)$ for which

$$
I_{q-1}<\Gamma\left(\lambda_{n}\right) \leqq I_{q} .
$$

(See schematic diagram in fig. 16.) Then, since $\Gamma(\lambda)$ is approximately linear and hence monotonic in the $n$th interval, the number $\gamma_{n}$ of roots in the $n$th interval is given by

$$
\gamma_{n}=\left|\mu_{n}-\mu_{n-1}\right| \text {. }
$$

The end points $\lambda_{n}$ can also be roots and must therefore be checked. We shall denote the $\gamma_{n}$ roots in the $n$th interval by

$$
\lambda_{n}{ }^{(j)} \quad j=1,2, \ldots, \gamma_{n}
$$

where

$$
\lambda_{n-1}<\lambda_{n}{ }^{(1)}<\lambda_{n}{ }^{(2)}<\ldots<\lambda_{n}{ }^{\left(\gamma_{n}\right)}<\lambda_{n}
$$
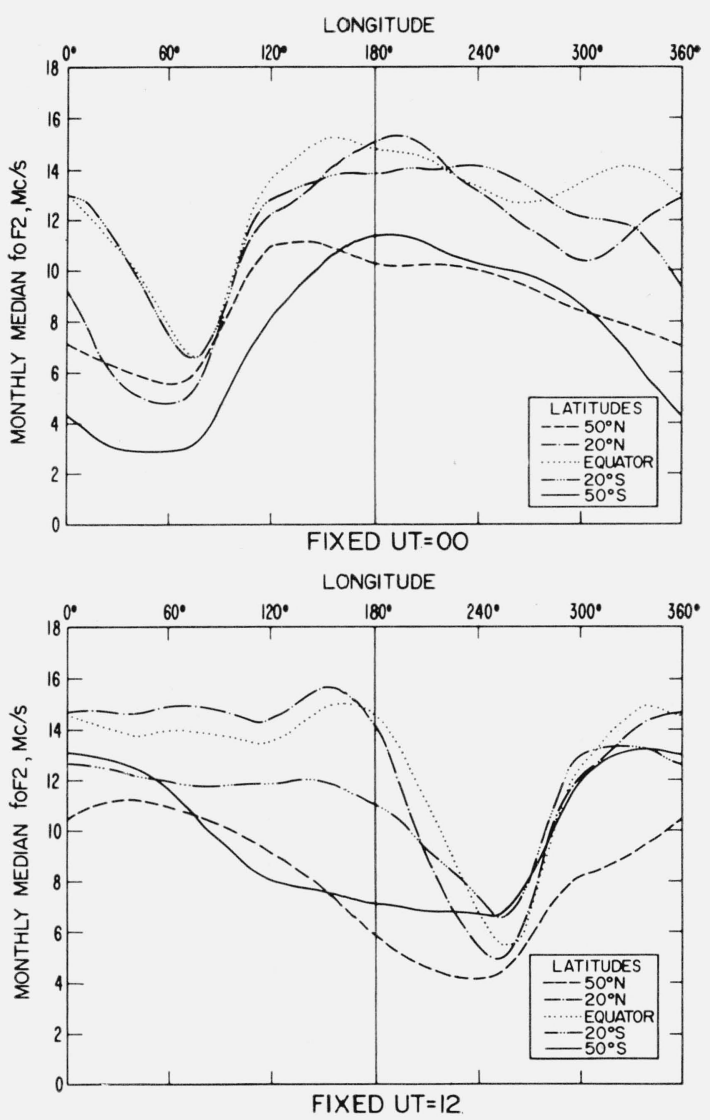

Figure 14. Longitudinal variation of monthly median $\mathrm{f}_{0} \mathrm{~F}_{2}$ from the function $\boldsymbol{\Gamma}(\boldsymbol{\lambda}, \theta, \boldsymbol{t})$ for September 1958.

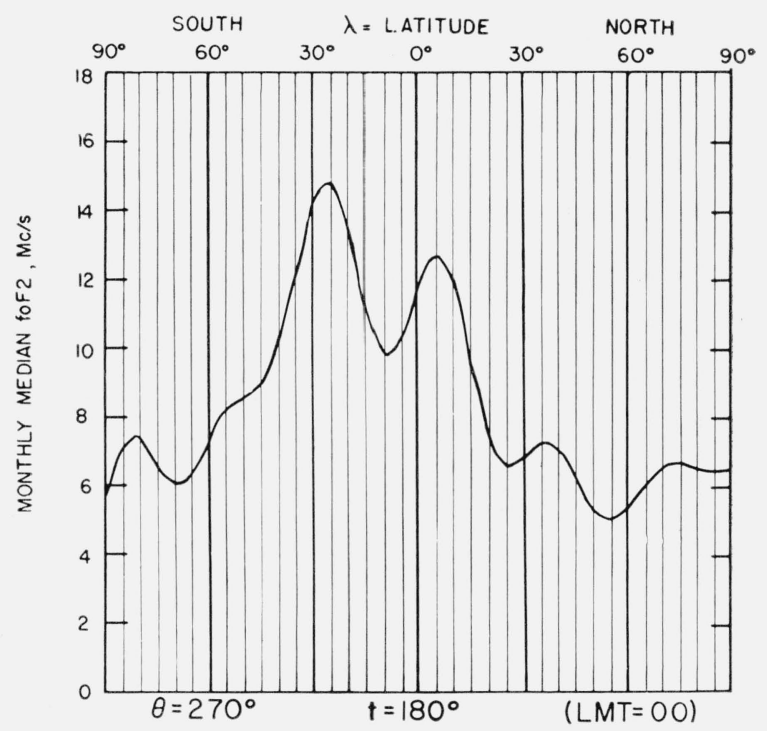

Figure 15. Latitudinal variation of monthly median $\mathrm{f}_{0} \mathrm{~F}_{2}$ from the function $\Gamma(\lambda, \theta, t)$ for September 1958. 
and for simplicity we shall write

$$
\Gamma_{n}^{(j)}=\Gamma\left(\lambda_{n}{ }^{(j)}\right) \quad j=1,2, \ldots, \gamma_{n} .
$$

Again, from the monotonic behavior of $\Gamma(\lambda)$ in the $n$th interval it can be seen that

(A) $\mu_{n-1}<\mu_{n}$ implies $\Gamma_{n}^{(j)}=I_{\mu_{n-1}+j-1}$

(B) $\mu_{n-1}>\mu_{n}$ implies $\Gamma_{n}(j)=1,2, \gamma_{n}$

(C) $\mu_{n-1}=\mu_{n}$ implies there are no solutions in the $n$th interval.

Thus we have determined the number of roots in the $n$th interval as well as the values assumed by $\Gamma(\lambda)$ at these roots. The final problem of approximating each root successively is resolved in the following section.

\subsection{Method of Successive Approximation}

Since $N$ was so chosen that the function $\Gamma(\lambda)$ is approximately linear within any of the intervals (18), a satisfactory approximation can be made to the roots $\lambda_{n}{ }^{(1)}, \lambda_{n}{ }^{(2)}, \ldots ., \lambda_{n}{ }^{\left(\gamma_{n}\right)}$ in the $n$th interval by means of linear interpolation. In the procedure to be followed the size of the interval of interpolation is reduced after each approximation so that rapid convergence is assured.

For the first root $\lambda_{n}{ }^{(1)}$ the initial interval of interpolation will be $\left(a_{1}{ }^{(1)}, b_{1}{ }^{(1)}\right)$ where

$$
a_{1}^{(1)}=\lambda_{n-1} \text { and } b_{1}^{(1)}=\lambda_{n},
$$

so that the first approximation to $\lambda_{n}{ }^{(1)}$ is given by

$$
d_{1}^{(1)}=\frac{b_{1}^{(1)}\left[\Gamma_{n}^{(1)}-\Gamma\left(a_{1}^{(1)}\right)\right]-a_{1}^{(1)}\left[\Gamma_{n}^{(1)}-\Gamma\left(b_{1}^{(1)}\right)\right]}{\Gamma\left(b_{1}^{(1)}\right)-\Gamma\left(a_{1}^{(1)}\right)} .
$$

(See schematic diagram in fig. 16.) In general, the $r$ th approximation to the $j$ th root $\lambda_{n}{ }^{(j)}$ is given by

$$
d_{r}^{(j)}=\frac{b_{r}^{(j)}\left[\Gamma_{n}^{(j)}-\Gamma\left(a_{r}^{(j)}\right)\right]-a_{r}^{(j)}\left[\Gamma_{n}^{(j)}-\Gamma\left(b_{r}^{(j)}\right)\right]}{\Gamma\left(b_{r}^{(j)}\right)-\Gamma\left(a_{r}^{(j)}\right)}
$$

where $\left(a_{r}{ }^{(j)}, b_{r}{ }^{(j)}\right)$ denotes the $r$ th interval of interpolation for the $j$ th root (to be defined below). After each approximation we must determine which of the following cases occurs:

$$
\begin{aligned}
& \left|\Gamma_{n}^{(j)}-\Gamma\left(d_{r}^{(j)}\right)\right| \leqq \epsilon, \\
& \Gamma\left(a_{r}^{(j)}\right)<\Gamma_{n}^{(j)}<\Gamma\left(d_{r}^{(j)}\right) \leqq \Gamma\left(b_{r}^{(j)}\right), \\
& \Gamma\left(a_{r}^{(j)}\right) \leqq \Gamma\left(d_{r}^{(j)}\right)<\Gamma_{n}^{(j)}<\Gamma\left(b_{r}^{(j)}\right), \\
& \Gamma\left(a_{r}^{(j)}\right)>\Gamma_{n}^{(j)}>\Gamma\left(d_{r}^{(j)}\right) \geqq \Gamma\left(b_{r}^{(j)}\right), \\
& \Gamma\left(a_{r}^{(j)}\right) \geqq \Gamma\left(d_{r}^{(j)}\right)>\Gamma_{n}^{(j)}>\Gamma\left(b_{r}^{(j)}\right) .
\end{aligned}
$$

We note that (32) and (33) cannot occur if (25A) holds, and (30) and (31) cannot occur if (25B) holds. If (29) occurs, then clearly we have the

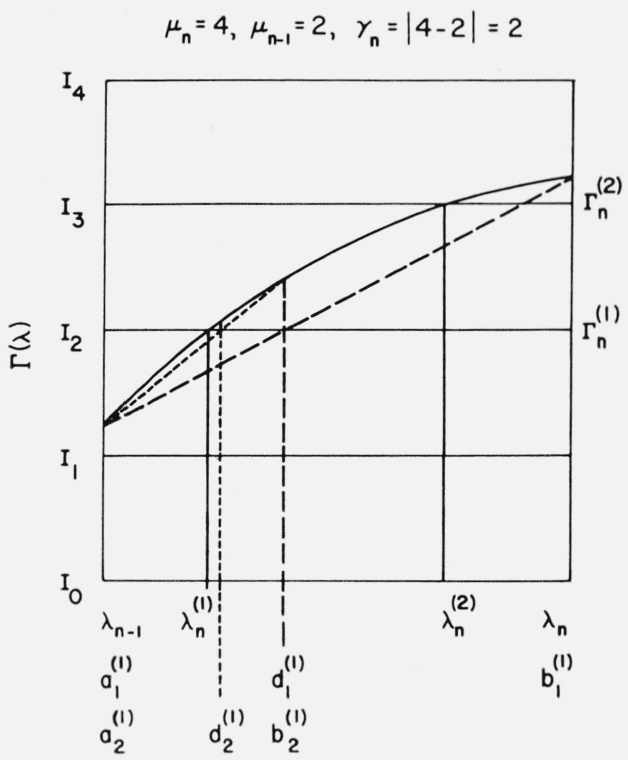

Figure 16. Schematic diagram of typical nth interval.

desired approximation. If (30) or (32) occur, then we let

$$
a_{r+1}^{(j)}=a_{r}^{(j)} \text { and } b_{r+1}^{(j)}=d_{r}^{(j)},
$$

whereas if (31) or (33) occur, we let

$$
a_{r+1}^{(j)}=d_{r}^{(j)} \text { and } b_{r+1}^{(j)}=b_{r}^{(j)}
$$

so that in any case the size of the new interval of interpolation is less than that of the preceding one. This process is continued until (29) occurs and we proceed to the next root. For each root $\lambda_{n}{ }^{(j)}$ after the first $\left(1<j \leqq \gamma_{n}\right)$, the initial interval of interpolation is taken to be

$$
a_{1}^{(j)}=\lambda_{n}^{(j-1)} \text { and } b_{1}^{(j)}=\lambda_{n}, j=2,3, \ldots, \gamma_{n}
$$

so that the convergence becomes faster for each successive root since the initial intervals are successively reduced.

The authors gratefully acknowledge the assistance they received from a number of persons. The examples of applications of "numerical maps," which illustrate the methods given in the present paper, were rendered possible by the careful work of Miss Martha Hinds and Miss Minadora PoKempner. They have been responsible for the preparation and analysis of the ionospheric data and for programing on large-scale computers. Direct assistance has also been received from Mrs. Estelle D. Powell, Mrs. Anna von Kreisler, Ronald P. Graham, Mrs. Gladys Waggoner, and operators in the Computing Laboratories of the National Bureau of Standards (in Boulder, Colo., and in Washington, D.C.). 


\section{References}

Byerly, W. E., Fourier series and spherical, cylindrical, and ellipsoidal harmonics, pp. 144-218 (Ginn and Company, Boston, 1893)

Jones, W. B., Atlas of Fourier coefficients of diurnal variation of $\mathrm{f}_{0} \mathrm{~F}_{2}$, NBS Tech. Note 142 (PB161643) (Apr. 1962).

Jones, W. B., and R. M. Gallet, Ionospheric mapping by numerical methods, J. Intern. Telecomm. Union, No. 12, 260-264 (Dec. 1960).
Jones, W. B., and R. M. Gallet, The representation of diurnal and geographic variations of ionospheric data by numerical methods, Telecommunication J. 29, No. 5, 129-149 (May 1962), and J. Research NBS 66D (Radio Prop.) No. 4, 419-438 (July-Aug. 1962).

Wright, J. W., L. R. Wescott, and D. J. Brown, Mean electron density variations of the quiet ionosphere 4-June 1959, NBS Tech. Note 49-4 (May 1961).

(Paper 66D6-225) 